\title{
Colossal magnetodielectric effect caused by magnetoelectric effect under low magnetic field
}

\author{
QIAN LIU, XIAO-BING BIAN, JIAN-PING ZHOU* and PENG LIU \\ School of Physics and Information Technology, Shaanxi Normal University, Xi' an 710062, P.R. China
}

MS received 30 October 2008; revised 24 January 2011

\begin{abstract}
The colossal magnetodielectric effect is reported in $\mathrm{Pb}(\mathrm{Zr}, \mathrm{Ti}) \mathrm{O}_{3} /$ Terfenol-D laminate composite under low magnetic field. When the composite is placed in an external a.c. magnetic field, magnetoelectric effect is produced, as a result, the dielectric properties of the $\mathrm{Pb}(\mathrm{Zr}, \mathrm{Ti}) \mathrm{O}_{3}$ is changed, i.e. magnetodielectric effect. Both the amplitude and resonance frequency change with the external magnetic field. The colossal magnetodielectric coefficient of $5 \times 10^{4} \%$ at low magnetic field of 20 Oe is achieved near the electromechanical resonance frequency.
\end{abstract}

Keywords. Composite materials; electrical properties; magnetodielectric effect; magnetoelectric effect.

\section{Introduction}

Recently, multiferroic materials have attracted much interest for their coexistence of ferroelectricity, ferromagnetism and ferroelasticity (Kimura et al 2003; Wang et al 2003; Hur et al 2004; Spaldin and Fiebig 2005; Eerenstein et al 2006; Weber et al 2006; Mamin et al 2007). The coupling between ferroelectric and ferromagnetic orders can produce some new effects, such as magnetoelectric (ME) and magnetodielectric (MD) effects. The MD effect is described as a change of dielectric constant in an applied magnetic field, i.e. MD = $\Delta \varepsilon(f) / \varepsilon(0, f) \times 100 \%=[\varepsilon(H, f)-\varepsilon(0, f)] / \varepsilon(0, f) \times$ $100 \%$, where $\varepsilon(H, f)$ and $\varepsilon(0, f)$ are the dielectric constants of the multiferroic materials at frequency $f$ in and out of the external magnetic field, respectively. The MD effects have been studied mainly on single phases, which show dielectric change under an external magnetic field (Kimura et al 2003; Hur et al 2004; Lorenz et al 2004; Weber et al 2006; Mamin et al 2007). The possibility of tuning the dielectric constant by external magnetic field open perspectives for the basic understanding of the multiferroic materials and for the design of devices.

However, as the history of multiferroic research indicates, multiferroic materials in a single phase are known to be extremely rare (Nicola 2000; Prellier et al 2005). And the distinct change in the dielectric constant commonly occurs near the magnetic phase transition under external magnetic field (Kimura et al 2003; Prellier et al 2005), similar to the colossal magnetoresistance (Weber et al 2006). The dielectric anomalies near the phase transition resulted from strong competition and interplay among the charge, orbital

*Author for correspondence (zhoujp@ snnu.edu.cn, zhoujp@tsinghua.org.cn) and spin degrees of freedom (Mamin et al 2007). The MD effect is percentage value of several decades, and is much higher near the insulator-metal transition (Rairigh et al 2007) or incommensurate-commensurate transition (Kimura et al 2003). But the magnetic field needed for producing the MD effect is commonly as high as several teslas (Kimura et al 2003; Hur et al 2004; Lorenz et al 2004; Weber et al 2006; Mamin et al 2007; Rairigh et al 2007). Alternatively, composites have stimulated much scientific and technological interest because the multiferroic properties are easily obtained by combining the individual ferroic phases. The ME coupling in composites is much stronger resulting from the product property of both the phases even at room temperature in comparison with the single phase (Nan et al 2008). Analogously, the MD effect should be strong in the composites. On the other hand, the MD effect can be achieved through a combination of magnetoresistance and the Maxwell-Wagner effects without true ME coupling (Catalan 2006). Could the ME coupling result in a MD effect? Magnetoelastoelectric equivalent circuits are used to understand the ME coupling in the laminate composites (Dong et al 2003). It is easily concluded that magnetostrictive component under external magnetic field could change the dielectric properties of the piezoelectric component from the equivalent circuits. Therefore, the ME effect can result in a MD effect. In this paper, we choose a simple $\mathrm{Pb}(\mathrm{Zr}, \mathrm{Ti}) \mathrm{O}_{3} /$ Terfenol-D laminate composite, in which a strong ME coupling is revealed (Zhou et al 2008), and study the colossal MD effect caused by the ME effect at much low a.c. magnetic field at room temperature.

\section{Experimental}

Terfenol-D and $\mathrm{Pb}(\mathrm{Zr}, \mathrm{Ti}) \mathrm{O}_{3}$ (PZT) were selected as magnetostrictive and piezoelectric components, respectively for 
their excellent magnetostrictive and piezoelectric properties. The PZT component was polarized along its thickness direction, $d_{33}$, of the PZT ceramic chip which is about $500 \mathrm{pC} / \mathrm{N}$. The transverse Terfenol-D strain $\left(\lambda_{\|}\right)$is $860 \mathrm{ppm}$ under the magnetic field of 700 Oe. The Terfenol-D and PZT tablets with the same diameter of $20 \mathrm{~mm}$ were bonded together by epoxy bonder. The thickness of the PZT and Terfenol-D was set to $1.5 \mathrm{~mm}$ and $3.5 \mathrm{~mm}$, respectively.

The capacitance variation with frequency $f_{\mathrm{E}}$ of the sample was measured with an Agilent E4980A impedance analyser. A signal generator drives a power amplifier, then a solenoid to generate an a.c. magnetic field, $H=H_{0} \cos 2 \pi f_{\mathrm{M}} t$, where $H_{0}$ and $f_{\mathrm{M}}$ are the amplitude and frequency of the a.c. magnetic field, respectively. The sample was kept in the uniform magnetic field at the solenoid centre while performing the dielectric measurement to obtain the magnetocapacitance. When the sample plane was parallel to the magnetic field, the transverse magnetodielectric sensitivity was obtained; and when the sample plane was perpendicular to the magnetic field, the longitudinal magnetodielectric sensitivity was measured.

\section{Results and discussion}

We first studied the transverse magnetodielectric effect for the composite. Under applied external a.c. magnetic field $H=H_{0} \cos 2 \pi f_{\mathrm{M}} t$ with $f_{\mathrm{M}}=10 \mathrm{kHz}$ and various amplitudes $H_{0}=0,1,2,3,4,6,8,12,16,20,24$ and $28 \mathrm{Oe}$, the dielectric constant of the composite was measured with an Agilent E4980A impedance analyser. The inset in figure 1(A) shows frequency $f_{E}$ dependence of dielectric constant, in which three electromechanical resonance modes appeared around $38 \mathrm{kHz}, 89.2 \mathrm{kHz}$ and $113.1 \mathrm{kHz}$, and are assigned to the first-order bending resonance mode, the second-order bending resonance mode and radial resonance mode, respectively (Shi et al 2007; Zhou et al 2008). Figure 1(A) shows the enlarged patterns around the first-order bending resonance frequency to reveal the effect of the external a.c. magnetic field on the dielectric constant, in which the resonance frequency redshift occurs and the dielectric constant at peaks decreases first and then increases with $H_{0}$, exhibiting like a saddle. Thus, colossal MD effect is clearly present. The representative MD coefficient was summed in figures 1(B)1(D), from which we know that the maximal MD can reach $104 \%$ when the magnetic amplitude $H_{0}$ is only $1 \mathrm{Oe}$. The maximal MD is as high as $5 \times 10^{4} \%$ at $H_{0}=20 \mathrm{Oe}$. There exists similar characteristics around the other two resonance peaks. We defined relative changes of dielectric resonance frequency and dielectric resonance peak amplitude as

$$
\begin{aligned}
R f_{\mathrm{E}} & =\Delta f_{\mathrm{E}} / f_{\mathrm{E}}(0) \times 100 \% \\
& =\left[f_{\mathrm{E}}(H)-f_{\mathrm{E}}(0)\right] / f_{\mathrm{E}}(0) \times 100 \%,
\end{aligned}
$$
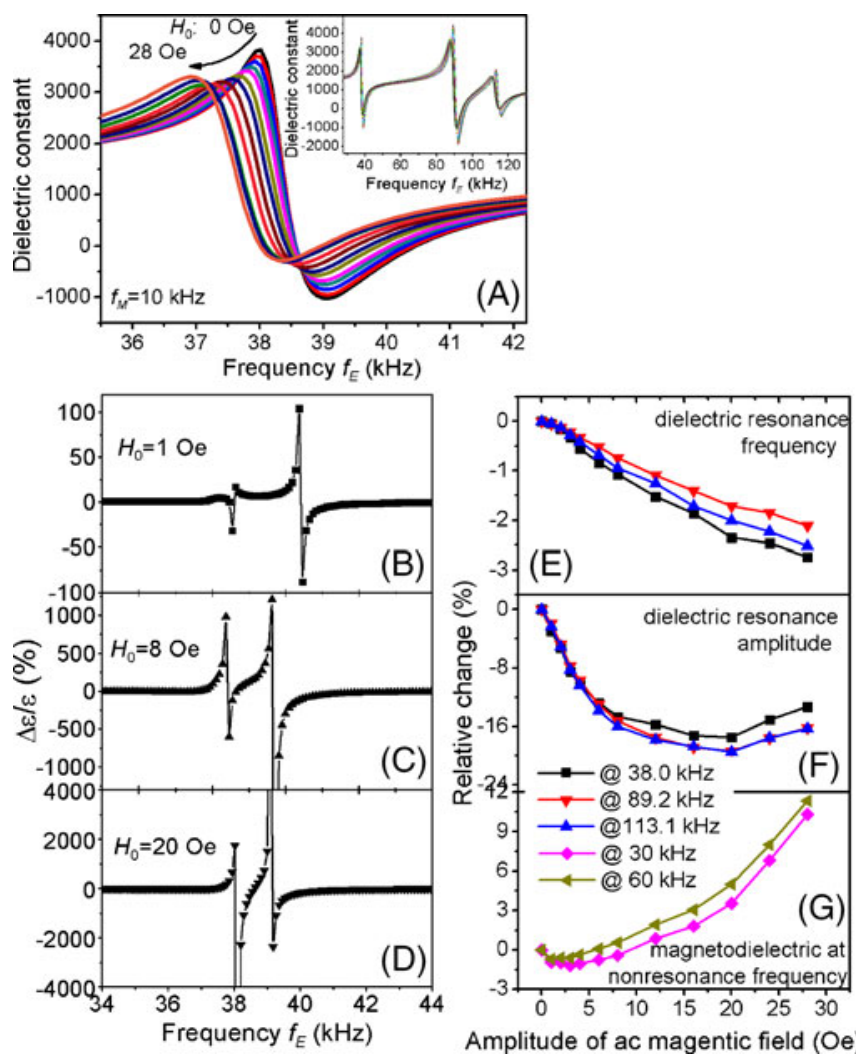

Figure 1. (A) Frequency $f_{E}$ dependence of dielectric constants for the composite near the first-order bending resonance frequency under a.c. magnetic field with $10 \mathrm{kHz}$ and various amplitudes parallel to the sample plane. The inset shows the corresponding dielectric constants in $28-130 \mathrm{kHz}$; (B)-(D) representative colossal magnetodielectric coefficients near the first-order bending resonance frequency under a.c. magnetic field with $10 \mathrm{kHz}$ and amplitudes of 1 , 8 and $20 \mathrm{Oe}$; (E) and (F) relative changes of resonance frequencies and resonance peak amplitudes with the a.c. magnetic field amplitude; (G) magnetodielectric coefficients at nonresonance frequencies of 30 and $60 \mathrm{kHz}$ dependence on the a.c. magnetic field amplitude.

$$
\begin{aligned}
R \varepsilon^{\prime} & =\Delta \varepsilon^{\prime} / \varepsilon^{\prime}(0) \times 100 \% \\
& =\left[\varepsilon^{\prime}(H)-\varepsilon^{\prime}(0)\right] \times 100 \%
\end{aligned}
$$

where $f(H)$ and $f(0)$ are electromechanical resonance frequencies of the composite in and out of the external magnetic field; $\varepsilon^{\prime}(H)$ and $\varepsilon^{\prime}(0)$ are electromechanical resonance peak amplitudes of the sample in and out of the magnetic field, respectively. Then we summarize these changes for the three resonance modes in figures $1(\mathrm{E})$ and $1(\mathrm{~F})$. The relative change of dielectric resonance frequency linearly decreases with increasing $H_{0}$, but the resonance amplitude represents U-shaped characteristics, i.e. decreases first and then increases. The relative change of the resonance amplitude reaches $19.5 \%$ at $H_{0}=20$ Oe even without any regard to 

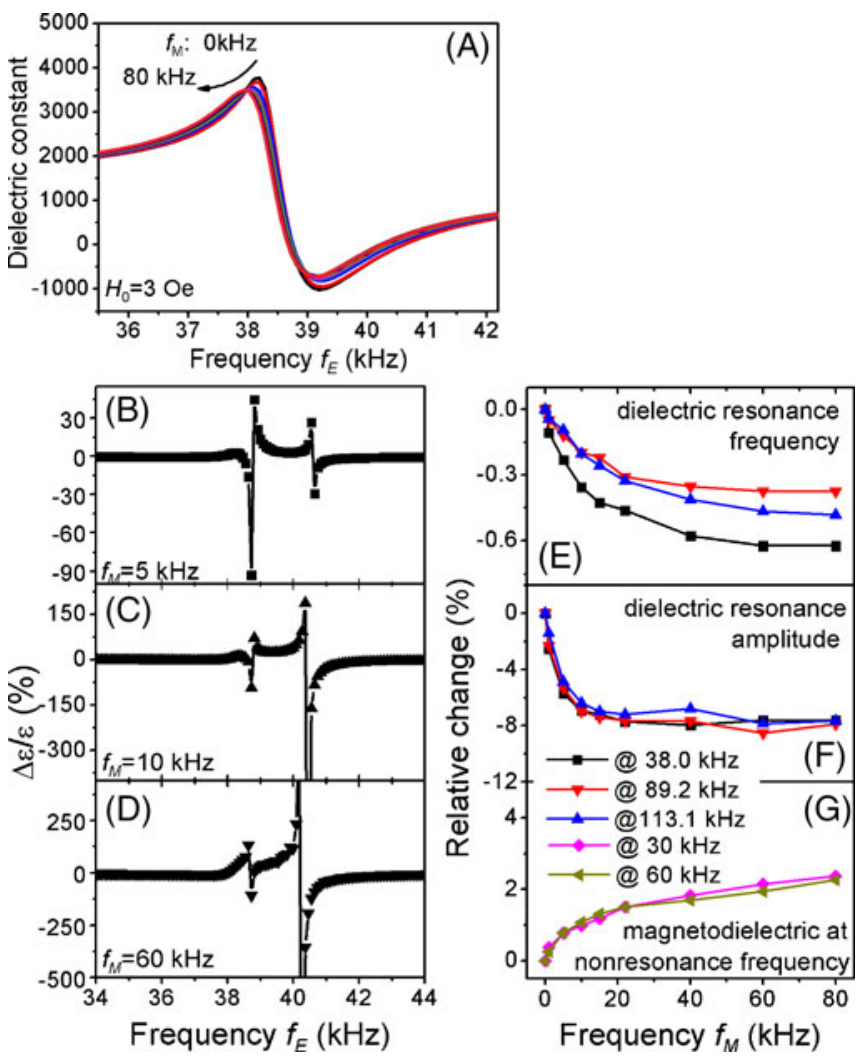

Figure 2. (A) Frequency $f_{\mathrm{E}}$ dependence of dielectric constants for the composite near the first-order bending resonance frequency under a.c. magnetic field with 3 Oe and various frequencies $f_{\mathrm{M}}$ parallel to the sample plane; (B)-(D) representative colossal magnetodielectric coefficients near the first-order bending resonance frequency under a.c. magnetic field with 3 Oe and frequencies of 5,10 and $60 \mathrm{kHz}$; (E) and (F) relative change of resonance frequency $f_{\mathrm{E}}$ and resonance peak amplitudes with the a.c. magnetic field frequency $f_{\mathrm{M}} ;(\mathrm{G})$ magnetodielectric coefficients at $f_{\mathrm{E}}=30$ and $60 \mathrm{kHz}$ dependence on the a.c. magnetic field frequency $f_{\mathrm{M}}$.

frequency. MD coefficients at nonresonance frequency of 30 and $60 \mathrm{kHz}$ are plotted in figure $1(\mathrm{G})$ for comparison. They decrease at first and then increase resulting from the characteristics in figure 1(A). The MD value reaches $10 \%$ at $H_{0}=$ $28 \mathrm{Oe}$. These values, even without considering the resonance frequency shift, are higher in comparison with the MD in single phase under much stronger magnetic field (Kimura et al 2003; Lorenz et al 2004).

Then we investigate the transverse MD effect under a.c. magnetic field with $H_{0}=3$ Oe and different frequencies $f_{M}=1,5,10,15,22,40,60$ and $80 \mathrm{kHz}$. Figure 2(A) shows the frequency $\left(f_{\mathrm{E}}\right)$ dependence of dielectric constant around the first-order bending resonance frequency. Colossal magnetodielectric effect is clearly present as shown in figures 2(B)-2(D) with the redshift of resonance frequency under a.c. magnetic field. The maximal MD reaches to $4 \times$ $10^{3} \%$ at $f_{\mathrm{M}}=15 \mathrm{kHz}$. Figures $2(\mathrm{E})$ and $2(\mathrm{~F})$ show the resonance frequency and amplitude changes with frequency

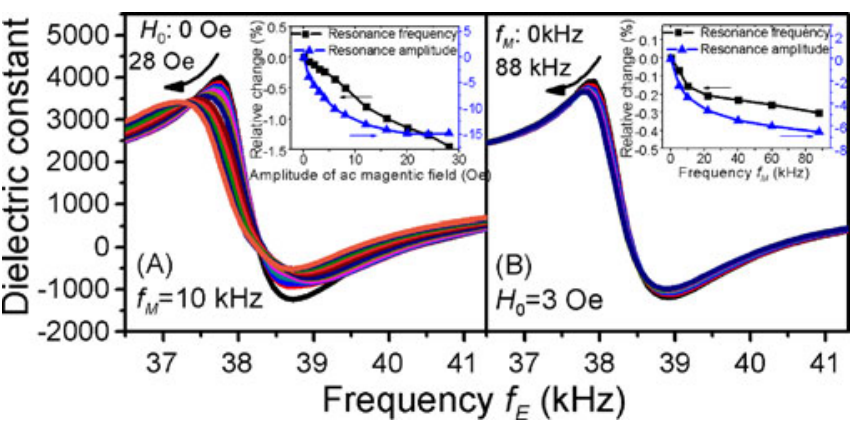

Figure 3. Frequency $f_{\mathrm{E}}$ dependence of dielectric constants for the composite near the first-order bending resonance frequency under external a.c. magnetic field: (A) with $f_{\mathrm{M}}=10 \mathrm{kHz}$ and various amplitudes, and (B) with $H_{0}=3$ Oe and various frequencies $f_{\mathrm{M}}$ perpendicular to the sample plane. The inset in (A) shows relative change of resonance frequency $f_{\mathrm{E}}$ and resonance peak amplitude with the a.c. magnetic field amplitude. The inset in (B) shows relative change of resonance frequency $f_{\mathrm{E}}$ and resonance peak amplitude with the a.c. magnetic field frequency $f_{\mathrm{M}}$.

$f_{\mathrm{M}}$ of the a.c. magnetic field. The relative dielectric resonance frequencies and amplitudes decrease with increasing $f_{\mathrm{M}}$ first, and then become unchangeable. MD coefficients at non-resonance frequencies $f_{\mathrm{M}}$ of $30 \mathrm{kHz}$ and $60 \mathrm{kHz}$ increase with the magnetic field frequency and reach $2 \%$ at $f_{\mathrm{M}}=80 \mathrm{kHz}$ as shown in figure $2(\mathrm{G})$.

The longitudinal MD effect shares similar characteristics with the transverse MD effect as shown in figures 3(A) and 3(B). The resonance peaks redshift occur with both the amplitude and frequency of the external a.c. magnetic field. But the relative changes are smaller than the corresponding values for the transverse MD effect.

The MD effect is relative with the ME effect. The ME polarization increases with the magnetic field (Shi et al 2007; Nan et al 2008), resulting in an enhanced MD coefficient as shown in figures 1(B)-1(D) and figures 2(B)-2(D). The longitudinal ME coupling is weaker than the transverse ME coupling (Zhou et al 2008), leading to a weak longitudinal MD coefficient. When applying magnetic field on the ME composite, the polarization was induced on the surface of sample due to the ME effect. The polarization impacts the dielectric response and change dielectric constant. The dielectric constant or the capacitance of the composites can be adjusted by the low applied magnetic field.

\section{Conclusions}

In summary, although MD effect can be achieved without true ME coupling, ME coupling can result in colossal MD effect in $\mathrm{Pb}(\mathrm{Zr}, \mathrm{Ti}) \mathrm{O}_{3} /$ Terfenol-D laminate composite. The MD coefficients are sensitive to both the amplitude and frequency of the external a.c. magnetic field. The resonance 
frequency can be adjusted by an external a.c. magnetic field. The results are helpful for understanding the relationship between the MD and ME effects.

\section{Acknowledgement}

This work was supported by the Program for New Century Excellent Talents in University (Grant No. NCET-10-0536).

\section{References}

Catalan G 2006 Appl. Phys. Lett. 88102902

Dong S, Li J F and Viehland D 2003 IEEE Trans. Ultrason. Ferroelect. Freq. Contr. 501253

Eerenstein W, Mathur N D and Scott J F 2006 Nature 442759

Hur N, Park S, Sharma P A, Ahn J S and Cheong S W 2004 Nature 429392
Kimura T, Goto T, Shintani H, Ishizaka K, Arima T and Tokura Y 2003 Nature $\mathbf{4 2 6} 55$

Lorenz B, Wang Y Q, Sun Y Y and Chu C W 2004 Phys. Rev. B70 212412

Mamin R F, Egami T, Marton Z and Migachev S A 2007 Phys. Rev. B75 115129

Nan C W, Bichurin M I, Dong S, Viehland D and Srinivasan G 2008 J. Appl. Phys. 103031101

Nicola A H 2000 J. Phys. Chem. B104 6694

Prellier W, Singh M P and Murugavel P 2005 J. Phys.: Condens. Matter 17 R803

Rairigh R P, Singh-Bhalla G, Tongay S, Dhakal T, Biswas A and Hebard A F 2007 Nat. Phys. 3551

Shi Z, Ma J, Lin Y and Nan C W 2007 J. Appl. Phys. 101043902

Spaldin N A and Fiebig M 2005 Science 309391

Wang J et al 2003 Science 2991719

Weber S, Lunkenheimer P, Fichtl R, Hemberger J, Tsurkan V and Loidl A 2006 Phys. Rev. Lett. 96157202

Zhou J P, Meng L, Xia Z H, Liu P and Liu G 2008 Appl. Phys. Lett. 92062903 